\title{
Impacto dos Contratos Futuros do Ibovespa na Volatilidade dos Índices de Ações no Brasil: Uma Análise na Crise do Subprime
}

\author{
Leandro Maciel \\ Doutorando - Faculdade de Engenharia Elétrica e Computação (DCA-FEEC/UNICAMP) \\ Endereço para contato: Av. Albert Einstein, 400 - Barão Geraldo - Campinas - São Paulo \\ CEP: 13083-852 - E-mail: maciel@dca.fee.unicamp.br
}

\author{
Rodrigo Lanna Franco da Silveira \\ Professor - Instituto de Economia (IE-UNICAMP) \\ Endereço para contato: Rua Pitágoras, 353 - Barão Geraldo - Campinas - São Paulo \\ CEP: 13083-857 - Email: rodrigolanna@eco.unicamp.br
}

\section{Ivette Luna}

Professora - Instituto de Economia (IE-UNICAMP)

Endereço para contato: Rua Pitágoras, 353 - Barão Geraldo - Campinas - São Paulo

CEP: 13083-857 - E-mail: ivette@eco.unicamp.br

\section{Rosangela Ballini}

Professora - Instituto de Economia (IE-UNICAMP)

Endereço para contato: Rua Pitágoras, 353 - Barão Geraldo - Campinas - São Paulo

CEP: 13083-857 - E-mail: ballini@eco.unicamp.br

Recebido em 17 de maio de 2011 . Aceito em 03 de julho de 2012.

\section{Resumo}

O aumento das negociações de derivativos no mercado mundial tem levado a um amplo debate acerca da influência dos contratos futuros sobre os preços à vista em diferentes mercados. Neste contexto, o presente artigo teve por objetivo avaliar, no período da crise do subprime, a influência da volatilidade dos preços futuros do IBOVESPA sobre os seguintes índices à vista: IBOVESPA, FGV-100, IBrX-50, IGC, SMLL e MLCX. Considerou-se o período entre agosto de 2007 e abril de 2009, quando as evidências da crise foram mais intensas até a retomada de crescimento dos índices acionários. Para se avaliar a causalidade na variância, foram empregados testes propostos por Cheung e Ng (1996) e Hafner e Herwartz (2006), sendo a volatilidade estimada por um processo GARCH univariado. Os resultados levaram à rejeição da hipótese de que, durante a crise do subprime, os movimentos do mercado futuro desestabilizaram o mercado à vista de ações brasileiro.

\section{Palavras-Chave}

mercados futuros, índice de ações, causalidade, volatilidade

- Os autores agradecem as sugestões dos participantes do X Encontro Brasileiro de Finanças e pelos comentários dos pareceristas anônimos. L. Maciel agradece a Capes pelo apoio financeiro. R. Ballini agradece ao CNPq e à FAPESP o apoio financeiro à pesquisa. 


\title{
Classificação JEL
}

G13, G32

\begin{abstract}
Significant increasing in derivatives trading over the world markets has led to an interesting debate about futures contracts influences on spot prices. In this context, this paper aims to evaluate, during the subprime crisis, the influence of IBOVESPA futures price volatility on the spot price indices as follows: IBOVESPA, FGV-100, IBrX-50, IGC, SMLL and MLCX. We considered the period from August 2007 to April 2009, when the evidence of the crisis were intense until to be recovering of growth of stock market index. To assess causality-in-variance, tests proposed by Cheung and $\mathrm{Ng}$ (1996) and Hafner and Herwartz (2006) were employed, and the volatility was estimated by an univariate GARCH process. It was found that the volatility of IBOVESPA futures contract did not destabilize spot indices during the subprime crisis.
\end{abstract}

\section{Keywords}

futures market, equity indices, causality, volatility

\section{Introdução}

A negociação de contratos de derivativos nos mercados financeiros mundiais tem apresentado significativo crescimento ao longo da década de 2000. Conforme Acworth (2011), entre 1999 e 2010, o número de contratos negociados em bolsas passou de 2,4 bilhões para 22,30 bilhões, representando um aumento médio anual de aproximadamente 22\%. Em 2010, os papéis de maior liquidez foram os derivativos (futuros e opções) de ações e índices de ações, com participações de, respectivamente, $28,19 \%$ e $33,25 \%$ do número total de contratos transacionados no mundo. ${ }^{1}$

A partir deste contexto, acadêmicos, órgãos reguladores, police makers e agentes de mercado têm analisado qual o impacto da introdução e do crescimento das negociações de derivativos nos preços spot dos ativos subjacentes a estes contratos. O debate é bastante controverso, como pode ser observado a seguir. Três correntes de pensamento podem ser destacadas dentro deste tema. A primeira considera que a criação e o desenvolvimento dos mercados futuros levam a uma maior instabilidade dos preços à vis-

1 Dados do Bank for International Settlements (BIS) mostram, ainda, que os valores nocionais dos contratos negociados em mercado de balcão (swaps, termo e opções), em instituições financeiras com matriz nos países pertencentes ao G10, elevaram-se de US\$80,32 trilhões para US\$582,66 trilhões entre o final de 1998 e junho de 2010. 
ta, no sentido de aumentar a variabilidade (i.e. volatilidade) das cotações. A justificativa para tal observação se baseia na intensa atuação, nestes mercados, de um grupo de especuladores, denominados noise traders, que são caracterizados pelo domínio de um baixo grau de informação. Eles são atraídos pela alta alavancagem que os derivativos permitem obter a um custo relativamente pequeno, em relação aos negócios feitos no mercado à vista. Ao observarem a tendência dos preços, tais agentes assumem posições de compra ou de venda, que potencializam o movimento de aumento ou de queda das cotações futuras, sendo isso transmitido às cotações à vista, dado o mecanismo de arbitragem existente entre tais mercados (Figlewski, 1981; Stein, 1987; Spyrou, 2005).

A segunda linha de pensamento defende que este aumento da volatilidade dos preços à vista, com a introdução dos derivativos, não deve ser explicada pela atuação dos noise traders. Os mercados futuros, por possibilitarem a negociação dos contratos em verdadeiros leilões, seja no ambiente de viva-voz ou no sistema eletrônico, permitem que as informações fluam com mais rapidez, existindo um maior processamento e transmissão para o mercado à vista, o que resulta num incremento da volatilidade (Cox, 1976; Antoniou et al., 1998).

Já a terceira corrente aponta que o desenvolvimento dos mercados de derivativos não provoca uma elevação estatisticamente significativa da volatilidade das cotações à vista ou, ainda, em alguns casos, permite uma queda da variabilidade de tais preços. Contrariamente à primeira linha, defende-se que, pela possibilidade de atingir alta alavancagem e existir custo reduzido nas operações, os mercados de futuros e de opções atraem agentes muito bem informados, que antes negociavam nos mercados spot. Dessa maneira, a interferência dos noise traders passa a ser residual, o que torna os mercados mais eficientes, dada a existência de um fluxo de informações mais intenso. Consequentemente, as cotações à vista passam a ter maior estabilidade (Srinvasan \& Bhat, 2008).

A maior parte dos trabalhos desenvolvidos neste tema tem por base os derivativos sobre ações e sobre índices de ações de diferentes países. Análises recentes, da década de 2000, foram feitas em países como Estados Unidos (Dawson \& Staikoupas, 2009), Espanha (Illueca \& Lafuente, 2003; Illueca \& Lafuente, 2008; Pilar \& Rafael, 2002), Itália (Bologna, 2002), Grécia (Alexakis, 2007; Drimbetasetas et al., 2007; 
Spyrou, 2005), Turquia (Kasman \& Kasman, 2008), México (Zhong, 2004), Índia (Debasish, 2009; Tripathy et al., 2009; Srinvasan \& Bhat, 2008; Rao, 2007; Bandivadekar \& Ghosh, 2003), Malásia (Pow \& Poshakwale, 2004), Coreia (Ryoo \& Smith, 2004; Bae et al., 2009), Taiwan (Chiang, 2002) E Hong Kong (Yo, 2001).

Os resultados, em geral, estão de acordo com a terceira corrente acima destacada. Ou seja, a introdução e/ou crescimento da negociação dos derivativos não levam ao aumento da volatilidade dos preços no mercado spot. Dos trabalhos citados, apenas cinco deles constataram elevação da volatilidade - Bae et al. (2009); Rao (2007); Zhong (2004); Pow e Poshakwale (2004); Ryoo e Smith (2004). ${ }^{2}$

Deve-se salientar, no entanto, que os estudos apontados, assim como a formulação das três correntes de pensamento, têm base na análise da influência dos derivativos sobre o padrão de variabilidade das cotações à vista em períodos de relativa normalidade do mercado. Em geral, os trabalhos empíricos acima destacados investigam tal influência comparando a volatilidade dos preços spot antes e depois do início das negociações de contratos futuros ou de opções, assim como avaliando a causalidade entre o volume de negociação dos derivativos e a volatilidade das cotações à vista.

A análise nestes mercados ganha maior relevância em períodos de crise, como visto no final da década de 1980 (crash da Bolsa de Nova Iorque), em meados dos anos de 1990 (falência do Barings Bank e da Long-Term Capital Management - LTCM), no início de 2000 (fraudes contábeis de várias corporações norte-americanas) e na segunda metade de 2008 (crise do subprime). Especialmente nestes períodos, um aumento da volatilidade dos índices de ações reforça o argumento de que uma das razões para tal situação é a existência dos derivativos, os quais desestabilizam o preço spot (no sentido de causar maior variabilidade das cotações), acarretando situações de caos financeiro. Dessa forma, as demandas para aumento da regulação nestes mercados se tornam intensas.

No Brasil, contratos de derivativos de ações, índice de ações, taxas de câmbio, taxas de juros e commodities são negociados na Bolsa de Valores, Mercadorias e Futuros (BM\&FBOVESPA). Tal instituição

2 Outros trabalhos foram realizados em anos anteriores à década de 2000 e, da mesma maneira que as pesquisas acima citadas, grande parte delas não verificou elevação da variabilidade das cotações no mercado à vista (Mayew, 2000). 
foi considerada, em 2010, a sexta maior bolsa do mundo em volume de contratos negociados. No período 2000-2010, as transações e/ ou registros evoluíram de 82,95 milhões para 618,63 milhões de contratos, não considerando derivativos sobre ações individuais. Um dos importantes contratos negociados nesta bolsa consiste no futuro de IBOVESPA. Entre 2006 e 2010, este derivativo obteve uma participação média próxima a $5 \%$ do total de contratos negociados nesta bolsa.

Diante de tais considerações, o presente estudo tem o objetivo de avaliar, no período da crise do subprime, a influência da volatilidade dos preços futuros do IBOVESPA sobre seis índices à vista: IBOVESPA, FGV-100, IBrX-50 (Índice Brasil 50), IGC (Índice de Governança Corporativa), SMLL (Índice BM\&FBOVESPA Small Cap) e o MLCX (Índice BM\&FBOVESPA Mid Large Cap).

Vale notar que a análise enfatiza o período da crise do subprime, uma vez que, em momentos de pânico financeiro, as posições especulativas em derivativos tendem a ser liquidadas. Consequentemente, este movimento de reversão de posições leva a uma intensificação na volatilidade dos preços futuros, o que pode causar uma desestabilização no mercado à vista.

De forma a atingir o objetivo acima citado, o trabalho está dividido em três partes, além desta introdução. A seção seguinte descreve a metodologia adotada. Posteriormente, apresentam-se os resultados e sua discussão e, por fim, as conclusões e sugestões para trabalhos futuros.

\section{Metodologia}

\subsection{Amostra}

As séries, que representam o mercado brasileiro de ações à vista, serão compostas por dados diários dos Índices BOVESPA (IBOVESPA), FGV-100, Índice Brasil 50 (IBrX-50), Índice de Ações com Governança Corporativa Diferenciada (IGC), Índices BM\&FBOVESPA Small Cap (SMLL) e BM\&FBOVESPA Mid Large Cap (MLCX), enquanto que o mercado futuro será descrito pelo 
IBOVESPA Futuro. ${ }^{3}$ A amostra se inicia em 09/08/2007, dia em que o banco francês BNP Paribas surpreendeu os agentes de mercado ao bloquear temporariamente a retirada de três fundos devido à incapacidade de avaliação sobre seus ativos que estavam atrelados aos empréstimos de tipo subprime norte-americano. O final da amostra se dá em 30/04/2009, quando o mercado acionário iniciou um comportamento de retomada de crescimento. A Figura 1 apresenta as séries avaliadas.

3 Esses contratos têm como ativo objeto o IBOVESPA, com cotação em pontos do próprio índice, sendo cada ponto igual a $\mathrm{R} \$ 1,00$. 


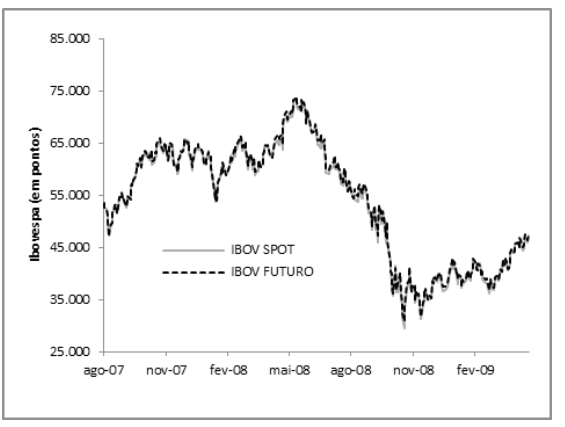

(a) Ibovespa

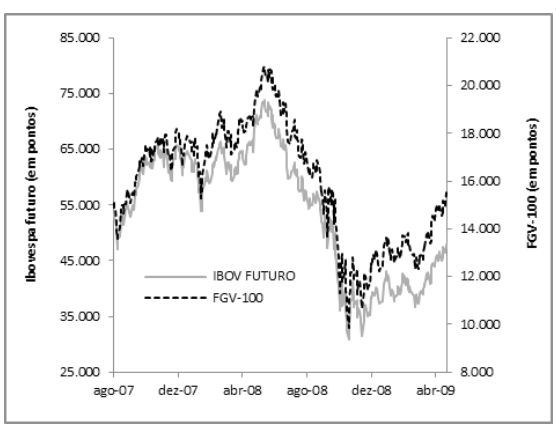

(c) FGV-100

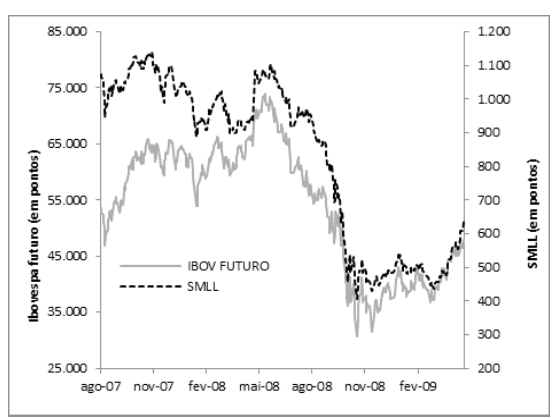

(f) SMLL

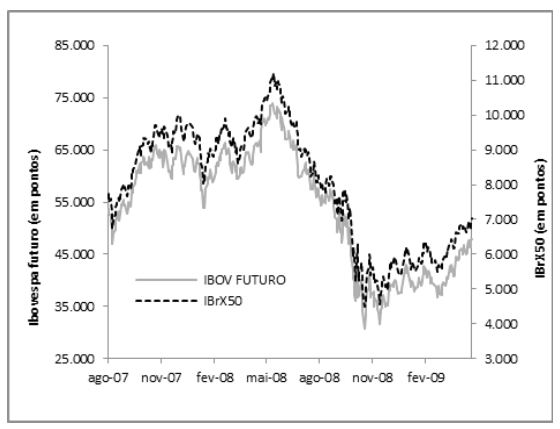

(b) $\operatorname{IBrX50}$

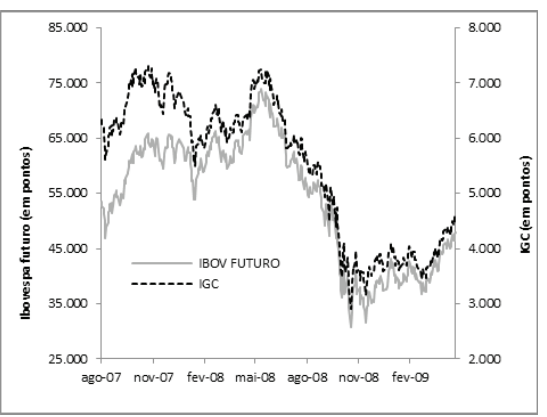

(e) IGC

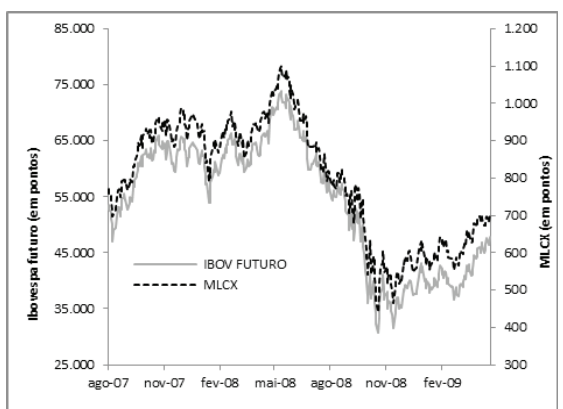

(g) MLCX

Figura 1 - Evolução das cotações futuras e à vista dos índices acionários considerados no estudo entre agosto de 2007 e abril de 2009

A escolha dos índices para o mercado spot teve base o fato de possuírem características diferentes em suas metodologias de cálculo e/ ou em sua carteira teórica. Dessa forma, torna-se possível verificar se os preços futuros do Ibovespa influenciaram o mercado de ações 
como um todo, não limitando a análise exclusivamente ao portfólio do Ibovespa, contemplando, inclusive, carteiras compostas por ações de menor liquidez.

O IBOVESPA corresponde ao mais tradicional índice de ações brasileiro, sendo este ponderado por liquidez, em que a participação de cada ação é determinada por um índice de negociabilidade que leva em conta o número de negócios e volume financeiro do respectivo papel em relação ao total observado no mercado. O Índice FGV-100 é uma importante referência de desempenho das ações de segunda linha, sendo sua carteira teórica formada por 100 empresas privadas não financeiras, com papéis negociados na BM\&FBOVESPA, selecionadas segundo critérios de excelência empresarial e liquidez. $\mathrm{O}$ IBrX-50, por sua vez, mede o retorno total de um portfólio teórico composto pelas 50 ações mais negociadas na BM\&FBOVESPA em termos de liquidez, ponderadas na carteira pelo seu valor de mercado. ${ }^{4}$ O IGC capta o desempenho de uma carteira teórica composta por ações de empresas que apresentam bons níveis de governança corporativa, sendo a ponderação dos papéis determinada pelo seu respectivo valor de mercado multiplicado por um fator de governança. Por fim, o SMLL e o MLCX medem o comportamento de empresas listadas na Bolsa de modo segmentado, em que o primeiro (segundo) tem foco nas corporações de menor (maior) capitalização. A seleção das ações segue critério de liquidez e a ponderação é feita pelo valor de mercado dos papéis disponíveis à negociação.

\subsection{Modelo GARCH}

Diversas técnicas são utilizadas para a estimação da volatilidade de séries financeiras, tais como: desvio padrão, alisamento exponencial (EWMA), modelos da família ARCH (Autoregressive Conditional Heteroscedasticity) e modelos de volatilidade estocástica. Estudos recentes mostram que os modelos GARCH ("Generalized ARCH") são os mais utilizados e apropriados para as séries em questão por se tratarem de uma forma simples e eficaz de modelar a incerteza sobre a média dos preços desses ativos (Galvão et al., 2000). Poon e Granger (2003), por exemplo, avaliam diversas técnicas de estimação da volatilidade em mercados financeiros e confirmam a adequação

4 Esse índice se mostra como um referencial para investidores e administradores de carteira, tendo a vantagem operacional de ser facilmente reproduzido pelo mercado. 
dos processos da família GARCH. Adicionalmente, Knight e Satchell (2007) consideram a estimação, previsão e aplicação das estimativas obtidas por modelos GARCH na precificação de contratos de opções, avaliação de riscos e otimização de portfólios, no qual aponta que tais modelos são capazes de capturar o padrão de variabilidade observado em séries financeiras. Além disso, recentemente, Drakos et al. (2010) analisaram, empiricamente, modelos GARCH e suas extensões na estimação da variabilidade no mercado de ações ateniense, enfocando o período da crise financeira mundial de 2007/2009. Os autores apontam que, mesmo em momentos de turbulências financeiras, os modelos GARCH são apropriados para a modelagem da volatilidade de retornos acionários. ${ }^{5}$ Neste sentido, neste artigo, será utilizado um modelo GARCH $(p, q)$ para obtenção da volatilidade dos índices de ações, de acordo com as equações:

$$
\begin{aligned}
& R_{t}=a+\sqrt{h_{t}} \varepsilon_{t} \\
& h_{t}=\alpha_{0}+\sum_{i=1}^{p} \alpha_{i} R_{t-1}^{2}+\sum_{j=1}^{q} \beta_{j} h_{t-j}
\end{aligned}
$$

em que, $R_{t}=\ln \left(\frac{P_{t}}{P_{t-1}}\right)$ indica a série dos retornos com base nos preços $\boldsymbol{P} ; \boldsymbol{a}$ é uma constante; $\boldsymbol{h}_{\boldsymbol{t}}$ representa a variância condicional de $\boldsymbol{R}_{\boldsymbol{t}} ; \boldsymbol{\varepsilon}_{\boldsymbol{t}}$ são erros, sendo estes independentes e identicamente distribuídos com média zero e variância unitária. Além disso, as condições de não negatividade da variância são tais que

$$
\alpha_{0}>0, \alpha_{i} \geq \mathbf{0}, \beta_{j} \geq \mathbf{0}, \sum_{i=1}^{r}\left(\alpha_{i}+\beta_{i}\right)<1, r=\max (p, q) \text {. }
$$

A modelagem da variância dos índices à vista e do IBOVESPA futuro será realizada de acordo com as Equações (1) e (2), tomando como única entrada seus retornos, capitalizados continuamente. Entretanto, como o vencimento do contrato futuro de IBOVESPA é toda quarta-feira mais próxima do dia 15 dos meses pares (fevereiro, abril, junho, agosto, outubro e dezembro), existe uma mudança do contrato de primeiro vencimento. Neste sentido, na modelagem da variância do IBOVESPA Futuro, será considerada uma variável dummy $(D)$, a qual assumirá valor igual a um nas datas de mudança

\footnotetext{
5 Para mais detalhes da estimação da volatilidade de ativos financeiros com modelos GARCH, ver Engle (2002), Laurent e Peters (2002), Alexander e Lazar (2003) e Alexander (2008).
} 
do contrato de primeiro vencimento e zero na demais datas. Assim, a expressão dos retornos para o IBOVESPA futuro seguirá:

$$
R_{t}=a+b \cdot D+\sqrt{h_{t}} \varepsilon_{t}
$$

em que $b$ é a constante associada à variável dummy. Deve-se notar que a expressão para a variância condicional mantém-se a mesma, conforme a Equação (2).

Os estimadores dos parâmetros dos modelos GARCH $(p, q)$ serão obtidos pelo método da máxima verossimilhança condicional. Com base na suposição de normalidade dos resíduos $\boldsymbol{\varepsilon}_{\boldsymbol{t}}$, define-se a $\log$ -verossimilhança, condicionada as $p$ primeiras observações, por:

$$
\mathcal{L}\left(\alpha, \beta \mid R_{1}, R_{2}, \cdots, R_{p}\right)=-\frac{1}{2} \sum_{t=p+1}^{N} \ln \left(h_{t}\right)-\frac{1}{2} \sum_{t=p+1}^{N} \frac{R_{t}^{2}}{h_{t}}
$$

sendo $N$ o número de observações utilizadas na estimação.

Em geral, a identificação da ordem de um modelo GARCH é uma tarefa difícil quando ajustado a uma série real. Morettin e Toloi (2006) recomendam a identificação de modelos de ordem baixa, como $(1,1),(1,2)$ ou $(2,1)$ para que, em seguida, seja aplicado algum critério de informação com a finalidade de se escolher o modelo mais adequado. Neste trabalho, o modelo GARCH será parametrizado de acordo com os critérios de informação Akaike (AIC), Schwarz (BIC) e Hannan-Quinn (HQC). O modelo escolhido será aquele que apresentar os menores critérios de informação, dado que estes penalizam modelos menos parcimoniosos (Akaike, 1973; Schwarz, 1978; Hannan \& Quinn, 1979).

\subsection{Testes de Causalidade}

Com o intuito de verificar a transmissão de informações entre os mercados futuro e à vista durante a crise do subprime, considerando a hipótese de que o mercado futuro desestabilizou o mercado spot, serão aplicados os testes baseados no conceito de causalidade de Wiener-Granger, desenvolvidos por Cheung e Ng (1996) e Hafner e Herwartz (2006). 
Granger (1969) propôs um teste de causalidade com base na assunção de que os eventos passados podem causar eventos atuais. Sejam duas séries temporais, $X_{t}$ e $Y_{t}$, estacionárias. O objetivo é verificar se a variação de $X$ "causa" a variação de $Y$, ou vice-versa. O teste de causalidade de Wiener-Granger assume que a informação relevante para a predição das respectivas variáveis $X$ e $Y$ está contida unicamente nos dados da série temporal dessas variáveis. Dessa forma, uma série de tempo estacionária $X$ causa, no sentido de Wiener-Granger, outra série estacionária $Y$ se melhores predições estatisticamente significantes de $Y$ podem ser obtidas ao se incluir valores defasados de $X$ às defasagens de $Y$. Com isso, desde que o futuro não possa predizer o passado, se a variável $X$ (Wiener-Granger) causa a variável $Y$, tem-se que mudanças em $X$ devem preceder temporalmente alterações em Y (Granger, 1969).

No estudo da relação de causalidade entre diferentes ativos e mercados, deve-se levar em conta as mudanças e os distúrbios em relação aos níveis de volatilidade, comumente descrito na literatura como "volatility spillovers" (HONG, 2001). Esses testes têm sido descritos na literatura com base em duas abordagens, como se observa a seguir.

O primeiro método foi desenvolvido por Cheung e Ng (1996). É baseado em um processo de dois estágios que avalia a função de correlação cruzada (FCC) dos quadrados dos resíduos padronizados, estimados por um modelo GARCH univariado. O primeiro estágio envolve a estimação de modelos univariados de série temporal que respondem a variações no tempo tanto na média condicional quanto na variância condicional. O segundo estágio, por sua vez, consiste na construção da série dos quadrados dos resíduos padronizados proveniente da variância condicional. A FCC do quadrado dos resíduos padronizados é usada para testar a hipótese nula de não causalidade na variância.

A partir das variâncias calculadas pelos modelos univariados, acima apresentados - Equações (1) e (2), obtém-se o quadrado dos resíduos padronizados das séries de mercados spot e futuro, $X_{t}$ e $Y_{t}$, respectivamente:

$$
u_{t}=\left[\frac{\left(X_{t}-\mu_{x, t}\right)^{2}}{h_{x, t}}\right]=\xi_{1}^{2} t
$$




$$
v_{t}=\left[\frac{\left(Y_{t}-\mu_{y, t}\right)^{2}}{h_{y, t}}\right]=\xi_{2}^{2} t
$$

sendo $\boldsymbol{\mu}_{\boldsymbol{x}, \boldsymbol{t}}$ e $\boldsymbol{\mu}_{\boldsymbol{y}, \boldsymbol{t}}$ os valores médios das séries de mercado spot e futuro, respectivamente, e $\boldsymbol{h}_{\boldsymbol{x}, \boldsymbol{t}}$ e $\boldsymbol{h}_{\boldsymbol{y}, \boldsymbol{t}}$ as variâncias condicionais dadas por (2). A correlação cruzada no instante $k, \boldsymbol{r}_{\boldsymbol{u}} \boldsymbol{v}(\boldsymbol{k})$, é dada por:

$$
r_{u v}=c_{u v}(k)\left(c_{u u}(0) c_{v v}(0)\right)^{-1 / 2}
$$

em que:

$c_{u v}(k)=N^{-1} \sum_{t=1}^{N}\left(u_{t}-\bar{u}\right)\left(v_{t}-\bar{v}\right) ; k=0, \pm 1, \pm 2, \cdots, c_{u u}(0)$

e $\boldsymbol{c}_{\boldsymbol{v} v}(\mathbf{0})$ são variâncias de $u$ e $v$, respectivamente; $T$ é o tamanho da amostra.

A partir de tais considerações, Cheung e $\mathrm{Ng}$ (1996) formularam um teste baseado na avaliação da significância da função de correlação cruzada (FCC) de $u$ e $v$ a fim de detectar relações causais e identificar a direção da causalidade em um segundo momento. A hipótese nula se baseia na não causalidade das variâncias estimadas. Para o teste de causalidade no instante $k$, compara-se $\sqrt{\boldsymbol{T}} \boldsymbol{r}_{\boldsymbol{u}}(\boldsymbol{k})$ com uma distribuição normal padronizada.

A segunda abordagem foi proposta por Hafner e Herwartz (2006). Este método tem base no princípio do Multiplicador Lagrangeano (ML). Considera-se um processo estocástico $\left\{\boldsymbol{\varepsilon}_{\boldsymbol{t}} \in \mathbb{R}^{\boldsymbol{n}}, \boldsymbol{t} \in \mathbb{N}\right\}$ estacionário, no espaço de probabilidade $(\Omega, F, P)$, e que $\boldsymbol{E}\left(\boldsymbol{\varepsilon}_{\boldsymbol{i t}} \mid \boldsymbol{F}_{\boldsymbol{t}-\mathbf{1}}\right)=\mathbf{0}$. O objetivo é testar a hipótese nula, $H_{0}$, para um dado $i, j=1, \ldots, n, i \neq j$ :

$H_{0}: \operatorname{Var}\left(\varepsilon_{i t} \mid F_{t-1}^{(j)}\right)=\operatorname{Var}\left(\varepsilon_{i t} \mid F_{t-1}\right)$

sendo, $\boldsymbol{F}_{\boldsymbol{t}-\mathbf{1}}^{(\boldsymbol{j})}=\boldsymbol{F}_{\boldsymbol{t}} \backslash \boldsymbol{h} \boldsymbol{\varepsilon}_{\boldsymbol{j} \boldsymbol{\tau}}, \boldsymbol{\tau} \leq \boldsymbol{t}$

Observa-se que a hipótese nula implica não causalidade na variância, ou seja, as informações da variância de outros processos estocásticos não afetam a variância do processo em questão. Para se testar tal hipótese, considera-se: 


$$
\varepsilon_{i t}=\xi_{i t} \sqrt{h_{i t}^{2} g_{t}}, g_{t}=1+z_{j t}^{\prime} \pi, z_{j t}=\left(\varepsilon_{j t-1}^{2}, h_{j t-1}^{2}\right)^{\prime}
$$

sendo $\boldsymbol{\xi}_{\boldsymbol{i} \boldsymbol{t}}$ o resíduo padronizado dado pelas Equações (5) e (6) e $\operatorname{Var}\left(\varepsilon_{i t} \mid \boldsymbol{F}_{t-1}\right)=\boldsymbol{\alpha}_{0}+\boldsymbol{\alpha}_{i} \boldsymbol{\varepsilon}_{i, t-1}^{2}+\boldsymbol{\beta}_{i} \boldsymbol{h}_{i t-1}^{2}$. O método utiliza, portanto, um modelo GARCH univariado, mais especificamente, um processo GARCH $(1,1)$.

Conforme Hafner e Herwartz (2006), analisando a Equação (9), uma condição suficiente para que a hipótese nula seja verdadeira é $\boldsymbol{\pi}=\mathbf{0}$. Dessa forma, as hipóteses podem ser reescritas como: $H_{0}: \pi=0$ e $H_{1}: \pi \neq 0$.

Os autores acima citados propuseram a estatística $\boldsymbol{\lambda}_{\boldsymbol{M} \boldsymbol{L}}$ para avaliar o teste proposto, ${ }^{6}$ sendo esta obtida mediante os seguintes passos: 1 ) estimação do GARCH $(1,1)$ e obtenção dos resíduos padronizados $\boldsymbol{\xi}_{\boldsymbol{i} \boldsymbol{t}}$, das derivadas $\boldsymbol{x}_{\boldsymbol{i} \boldsymbol{t}}$ e da volatilidade $\left.\boldsymbol{h}_{\boldsymbol{i} \boldsymbol{t}}^{\boldsymbol{2}} ; 2\right)$ regressão de $\boldsymbol{\xi}_{\boldsymbol{i} \boldsymbol{t}}^{\boldsymbol{2}}-\mathbf{1}$ contra $\boldsymbol{x}_{\boldsymbol{i}}^{\prime}$; 3) cálculo da estatística $\boldsymbol{\lambda}_{\boldsymbol{M}}$ mediante a multiplicação entre $N$ e o coeficiente de determinação $\left(R^{2}\right)$ da regressão no passo anterior. Se o valor da estatística superar o valor crítico (obtido a partir de uma distribuição $\boldsymbol{\chi}_{2}^{2}$ ), rejeita-se a hipótese nula de não causalidade na variância. O teste é feito de forma a avaliar a causalidade em ambos os sentidos, isto é, se a variância do futuro de Ibovespa "causa" a variância no índice à vista, e vice-versa.

\section{Resultados e Discussão}

A Figura 2 apresenta as séries consideradas em termos de retornos. Pode-se notar que as rentabilidades dos índices do mercado à vista foram muito próximas daquelas observadas para o IBOVESPA

$$
\lambda_{M L}=\frac{1}{4 T}\left[\sum_{t=1}^{T}\left(\zeta_{i t}^{2}-1\right) z_{j t}^{\prime}\right] V\left(\theta_{i}\right)^{-1}\left[\Sigma_{t=1}^{T}\left(\zeta_{i t}^{2}-1\right) z_{j t}\right]
$$

Sendo:

$$
V\left(\theta_{i}\right)=\frac{k}{4 N}\left[\sum_{t=1}^{N} z_{j t} z_{j t}^{\prime}-\sum_{t=1}^{N} z_{j t} x_{j t}^{\prime}\left(\sum_{t=1}^{N} x_{j t} x_{j t}^{\prime}\right)^{-1}\right] \sum_{t=1}^{N} x_{j t} z_{j t}^{\prime}
$$

Em que:

$$
\begin{aligned}
& k=\frac{1}{N} \sum_{t=1}^{N}\left(\xi_{i t}^{2}-1\right)^{2} \\
& x_{i t}=h_{i t}^{-2}\left(\partial h_{i t}^{2} / \partial \theta_{i}\right) \\
& \theta_{i}=\left(\alpha_{0}, \alpha_{i}, \beta_{i}\right)^{\prime} .
\end{aligned}
$$


Futuro. O SMLL foi o indicador que apresentou retornos que mais diferiram do IBOVESPA Futuro. Isso se explica pelas distintas composições dos portfólios dos índices.

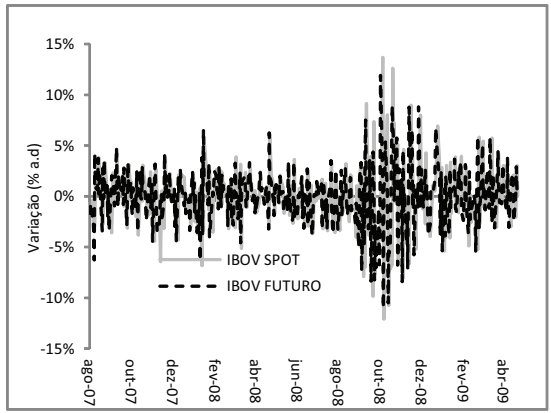

(a) Ibovespa

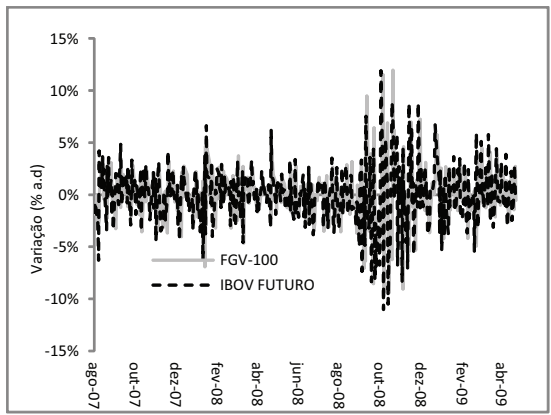

(b) FGV-100

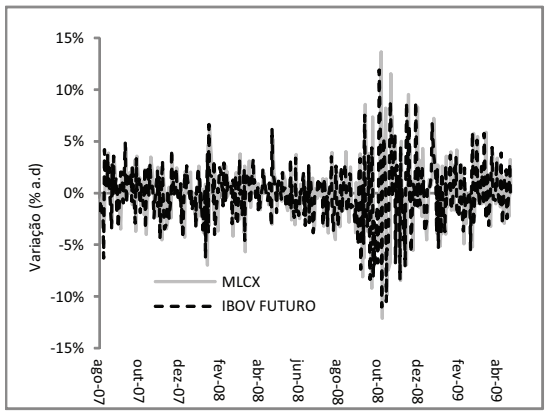

(f) MLCX

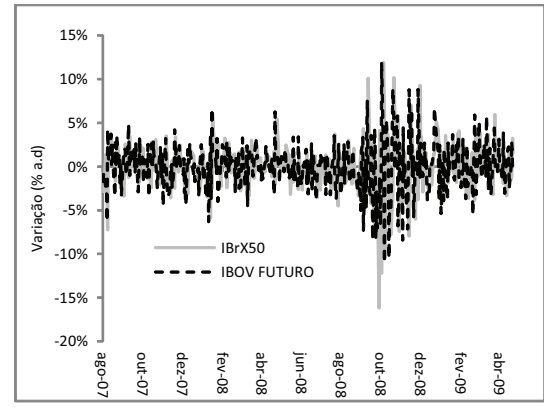

(c) IBrX50

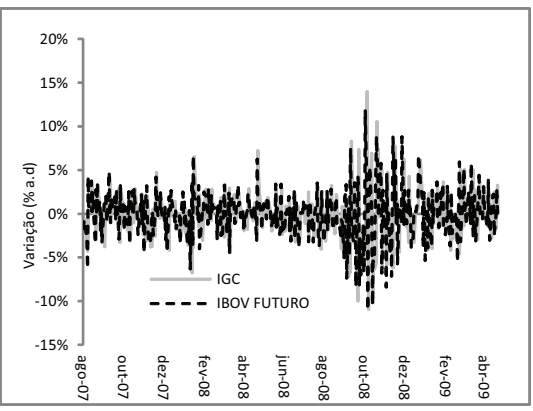

(d) IGC

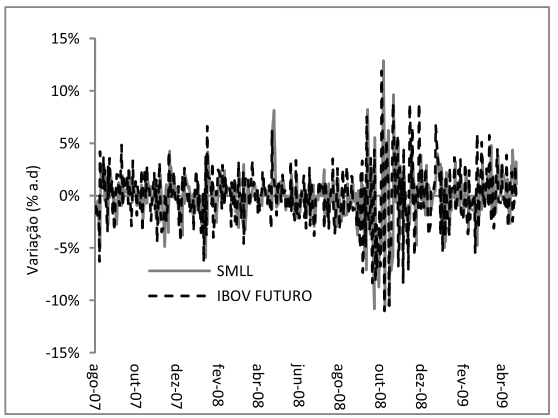

(e) SMLL

Figura 2 - Evolução dos retornos das cotações futuras e à vista dos índices acionários considerados no estudo entre agosto de 2007 e abril de 2009 
Todos os índices apresentaram a formação de um cluster nos retornos no início de setembro de 2008, que permaneceu até meados de janeiro de 2009. Essa oscilação maior no mercado de ações brasileiro se deveu ao fato de que em setembro de 2008 os agentes de mercado foram pouco receptivos às intervenções do Tesouro americano em favor do fortalecimento financeiro às agências Fannie Mae e Freddie Mac, que resultou na estatização dessas instituições. ${ }^{7}$ Corroborando esse fato, no mesmo mês, o pedido de concordata do banco de investimentos Lehman Brothers na Corte de Falências de Nova Iorque consolidou o aumento da tensão nos mercados financeiros internacionais. O pessimismo dos agentes ocasionou a chamada "corrida pela liquidez", em que as posições em mercados emergentes, ou avaliados de maior risco, foram desfeitas, o que explica as maiores oscilações nos retornos a partir de setembro. Ou seja, nos mercados de maior risco, como o Brasil, os agentes desfizeram suas posições para cumprir as obrigações referentes às perdas em seus mercados de origem. Dessa forma, a liquidez nos mercados emergentes reduziu-se drasticamente, ${ }^{8}$ resultando numa oscilação considerável, no sentido de queda no valor dos ativos desses mercados.

Na Tabela 1, verificam-se as estatísticas descritivas das séries dos retornos avaliadas neste trabalho. As médias das séries foram estatisticamente iguais a zero. Os desvios padrões foram muito próximos, sendo que os índices IBrX-50 e SMLL indicaram a maior e a menor volatilidade, respectivamente. Não se pode inferir, por meio dos dados da Tabela 1, que o mercado futuro foi mais ou menos volátil que o mercado à vista, mas nota-se que a série do mercado futuro apresentou características mais próximas da distribuição normal, segundo os coeficientes de assimetria e curtose, apesar de todas as séries não se distribuírem normalmente, de acordo com as estatísticas do teste

7 Essas duas instituições, em conjunto, administravam carteiras de ativos da ordem de US $\$ 5$ trilhões, cerca de $40 \%$ do total do mercado de hipotecas nos EUA e, com a crise, enfrentaram um grave problema de liquidez, o que levou o Tesouro americano realizar uma injeção de liquidez de US $\$ 200$ bilhões para tais instituições, para depois estatizá-las.

8 A quantidade de ações negociadas no mercado spot (lote padrão) da BM\&FBOVESPA, por exemplo, apresentou forte recuo no período da crise. Enquanto, entre 2005 e 2007, este mercado teve uma média anual de 4,73 trilhões de ações negociadas, no biênio 2008 e 2009, tal quantidade média passou para 3,59 trilhões/ano. Em 2010, o mercado teve forte recuperação, atingindo 8,35 trilhões de ações transacionadas. Dados do Banco Central do Brasil mostram que, no ano de 2008 (auge da crise), os investimentos estrangeiros em carteira tiveram uma saída líquida de US\$767 milhões (revertendo uma tendência positiva de entrada líquida de capitais desde 2005, chegando ao ápice de US\$48,1 bilhões em 2007). Adicionalmente, o volume financeiro líquido das transações feitas por investidores estrangeiros com ações de companhias brasileiras negociadas no país evoluiu de US $\$ 24,61$ bilhões para-US\$10,85 bilhões entre 2007 e 2008. 
Jarque-Bera (JB). Além disso, as séries dos retornos de todos os índices foram estacionárias, $I(0)$, segundo os testes de Dickey-Fuller Aumentado (ADF) e Phillips-Perron (P-Perron).

Tabela 1 - Característica das séries de retorno dos índices do mercado à vista $\mathrm{e}$ futuro

\begin{tabular}{|c|c|c|c|c|c|c|c|}
\hline Estatísticas & IBOV & FGV-100 & IGC & IbrX-50 & SMLL & MLCX & IBOV Futuro \\
\hline Média & $-0,00029$ & 0,00006 & $-0,00077$ & $-0,00022$ & $-0,00123$ & $-0,00020$ & $-0,00027$ \\
\hline Mediana & 0,00120 & 0,00146 & 0,00028 & 0,00181 & 0,00000 & 0,00183 & 0,00121 \\
\hline Máximo & 0,13678 & 0,11939 & 0,13973 & 0,13840 & 0,12888 & 0,13645 & 0,11899 \\
\hline Mínimo & $-0,12096$ & $-0,09414$ & $-0,10938$ & $-0,12885$ & $-0,10805$ & $-0,12101$ & $-0,11012$ \\
\hline Desv Pad & 0,02928 & 0,02561 & 0,02738 & 0,03063 & 0,02466 & 0,02940 & 0,02819 \\
\hline Assimetria & 0,07925 & 0,07537 & 0,17622 & 0,08103 & $-0,14125$ & 0,10307 & $-0,08647$ \\
\hline Curtose & 6,12120 & 6,29268 & 6,20736 & 5,68817 & 7,82430 & 5,70626 & 5,10383 \\
\hline Teste $\mathrm{JB}^{1}$ & 170,516 & 189,676 & 181,766 & 126,617 & 407,717 & 128,604 & 77,795 \\
\hline Teste ADF ${ }^{2}$ & $-20,935$ & $-21,405$ & $-20,215$ & $-20,436$ & $-18,585$ & $-20,601$ & $-20,930$ \\
\hline Teste P-Perron ${ }^{2}$ & $-21,371$ & $-21,993$ & $-20,401$ & $-20,798$ & $-18,551$ & $-21,002$ & $-21,264$ \\
\hline
\end{tabular}

${ }^{1}$ Para todas as séries avaliadas o teste Jarque-Bera indicou não normalidade às séries.

${ }^{2}$ As estatísticas foram significativas a $1 \%, 5 \%$ e $10 \%$.

A Tabela 2 apresenta os coeficientes de correlação entre os retornos dos índices avaliados. Verifica-se que os coeficientes estimados entre os retornos dos índices à vista e futuro foram todos próximos de um, indicando alta correlação. Assim, apesar das diferentes composições da carteira teórica de cada índice, os resultados permitem observar que, por se tratar de um período de crise financeira, os preços dos ativos transacionados no mercado acionário brasileiro possuem mesma evolução.

Tabela 2 - Correlação entre as séries dos retornos dos índices do mercado à vista e futuro

\begin{tabular}{lccccccc}
\hline & IBOV & FGV-100 & IGC & IbrX-50 & SMLL & MLCX & IBOV Futuro \\
IBOV & 1 & - & - & - & - & - & - \\
FGV-100 & 0,975 & 1 & - & - & - & - & - \\
IGC & 0,980 & 0,954 & 1 & - & - & - & - \\
IbrX-50 & 0,988 & 0,968 & 0,971 & 1 & - & - & - \\
SMLL & 0,904 & 0,888 & 0,922 & 0,872 & 1 & - & - \\
MLCX & 0,989 & 0,970 & 0,974 & 0.999 & 0,875 & 1 & - \\
IBOV Futuro & 0,961 & 0,943 & 0,954 & 0,956 & 0,890 & 0,957 & 1 \\
\hline
\end{tabular}

Conforme mencionado na Seção 2, estimou-se um processo GARCH de diferentes ordens, ${ }^{9}$ computando-se os valores dos critérios de informação AIC, BIC e HQC. Com base em tais critérios, determinou-se a ordem do modelo GARCH $(p, q)$ para as séries avaliadas,

9 Foram realizadas estimações de modelos com ordem variando de $(1,1)$ a $(8,8)$.

Est. Econ., São Paulo, vol. 42, n.4, p. 801-825, out.-dez. 2012 
de acordo com as Equações (1) e (2) para os índices à vista e as Equações (2) e (3) para o IBOVESPA futuro. Para todas as séries de retornos, os critérios de informação indicaram o processo GARCH $(1,1)$. Como a análise se dá durante a crise financeira mundial de 2007/2008, tais resultados indicam que as volatilidades dos índices apresentaram uma estrutura de dependência temporal similar e com memória bastante curta, em termos de retornos e variâncias passadas. A seguir, na Tabela 3, apresentam-se as estimações dos modelos GARCH para as séries consideradas.

Tabela 3 - Estimativas dos modelos GARCH $(1,1)$ univariados

\begin{tabular}{c|cc|cc}
\hline & \multicolumn{2}{|c|}{ IBOVESPA } & \multicolumn{2}{c}{ FGV -100 } \\
\hline Parâmetros & Estimação & Erro Padrão & Estimação & Erro Padrão \\
\hline$a$ & 0,00051 & 0,01126 & 0,00075 & 0,00101 \\
$a_{0}$ & 0,00002 & 0,00000 & 0,00001 & 0,00000 \\
$a_{1}$ & 0,09149 & 0,02602 & 0,09292 & 0,02422 \\
$\beta_{1}$ & 0,87960 & 0,03162 & 0,87898 & 0,02995 \\
\hline \multicolumn{2}{|c|}{ IBrX-50 } & \multicolumn{2}{c}{ IGC } \\
\hline Parâmetros & Estimação & Erro Padrão & Estimação & Erro Padrão \\
\hline$a$ & 0,00059 & 0,00120 & 0,00012 & 0,00111 \\
$a_{0}$ & 0,00001 & 0,00001 & 0,00002 & 0,00001 \\
$a_{1}$ & 0,08809 & 0,02491 & 0,09037 & 0,02903 \\
$\beta_{1}$ & 0,88886 & 0,02911 & 0,87038 & 0,03996 \\
\hline \multicolumn{5}{|c|}{ SMLL } \\
Parâmetros & Estimação & Erro Padrão & Estimação & MLCX \\
\hline$a$ & 0,00009 & 0,00094 & 0,00055 & 0,00116 \\
$a_{0}$ & 0,00003 & 0,00000 & 0,00001 & 0,02492 \\
$a_{1}$ & 0,20264 & 0,04868 & 0,08753 & 0,02492 \\
$\beta_{1}$ & 0,73828 & 0,04673 & 0,88803 & 0,02995 \\
\hline \multicolumn{2}{|c|}{ IBOV Futuro } & & \\
\hline Parâmetros & Estimação & Erro Padrão & & \\
\hline$a$ & 0,00044 & 0,00116 & & \\
$b$ & 0,00326 & 0,00584 & & \\
$a_{0}$ & 0,00001 & 0,00000 & & \\
$a_{1}$ & 0,08433 & 0,02459 & &
\end{tabular}

As estimativas dos coeficientes para o processo da volatilidade dos índices foram muito próximas, tanto para o mercado spot quanto para o mercado futuro, indicando um mesmo padrão de influência tanto dos retornos quanto da variância passados. Entretanto, para o Índice SMLL, o coeficiente do retorno defasado $(0,20264)$ foi maior que nos demais indicadores do mercado à vista e futuro, o que mostra uma maior persistência dos efeitos dos retornos passados para a determinação da volatilidade. Os valores estimados das variâncias dos índices do mercado à vista em comparação com o mercado futuro, em termos percentuais ao ano, estão representados na Figura 3. 
Por meio da avaliação da Figura 3, notam-se, em geral, dois clusters ou choques de volatilidade para o período analisado. O primeiro cluster teve início em novembro de 2007, sofrendo oscilações até abril de 2008. Esse fato se explica pelo desencadear inicial da crise do subprime, que não afetou de forma tão significativa os mercados de ações nas economias emergentes. Nesse período, os investidores internacionais desfizeram suas posições em créditos hipotecários, principalmente nos títulos de curto prazo (lastreados por ativos) nos EUA (commercial papers). Dessa forma, para honrar as perdas com as inversões referenciadas nos títulos imobiliários, os agentes sacaram suas aplicações nos mercados considerados de maior risco.

O segundo choque, de maior magnitude, ocorreu no início do mês de setembro de 2008, prolongando-se até meados de janeiro de 2009. Houve um aumento significativo na volatilidade dos índices de ações aqui estudados, o que mostra o momento crítico da crise do subprime na economia brasileira. Com o pedido de concordata do Lehman Brothers e a estatização das instituições Fannie Mae e Freddie Mac, um forte pânico se instaurou nos mercados globais. Os investimentos estrangeiros em ações de empresas brasileiras negociadas no País registraram, entre janeiro e maio de 2008, uma entrada líquida de cerca de US\$5,44 bilhões, conforme dados do BACEN. Em junho do mesmo ano, iniciou-se uma inversão do fluxo, totalizando, entre junho de 2008 e fevereiro de 2009, uma saída líquida de US\$17,14 bilhões, com destaque para o mês de outubro de 2008, quando US $\$ 6,07$ bilhões (em termos líquidos) deixaram o mercado acionário nacional. Somente em março de 2009, houve uma retomada dos ingressos líquidos de investimento estrangeiro em ações, igual a US\$843,9 milhões. 


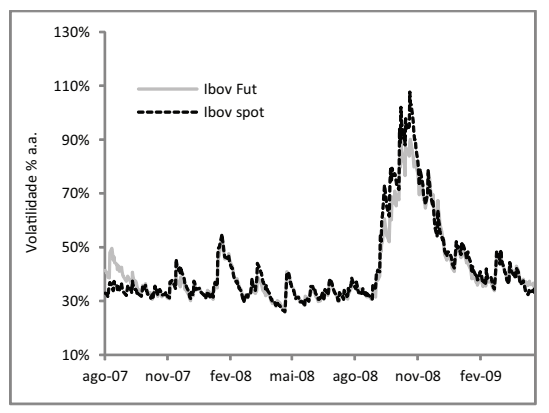

(a) Ibovespa

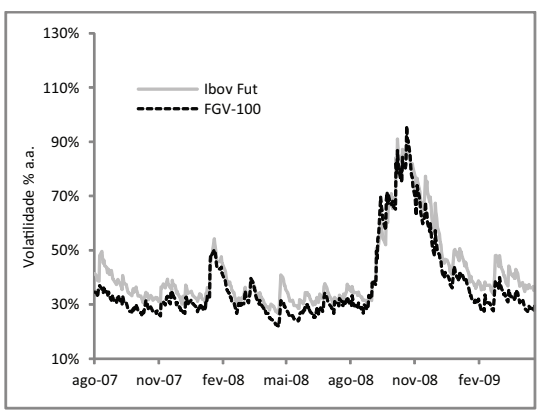

(c) FGV-100

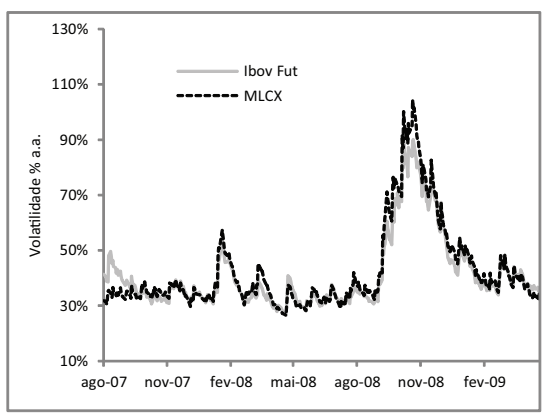

(e) MLCX

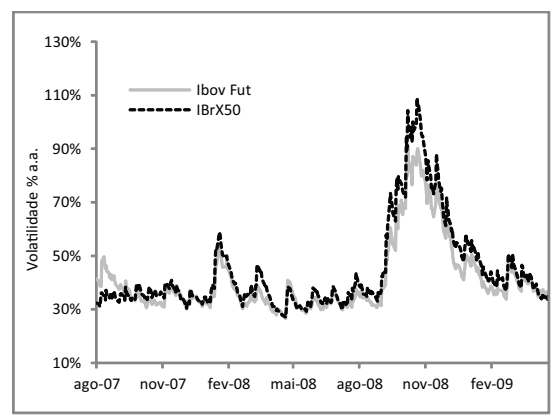

(b) $\operatorname{IBrX50}$

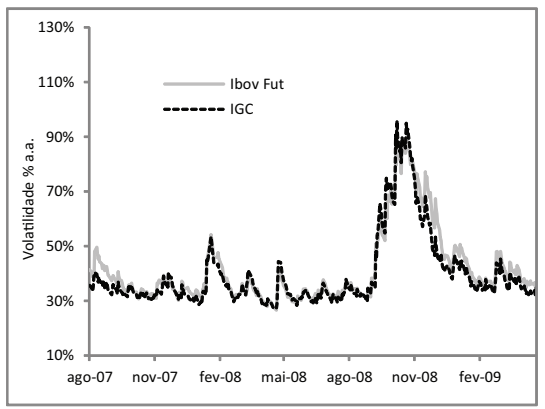

(d) IGC

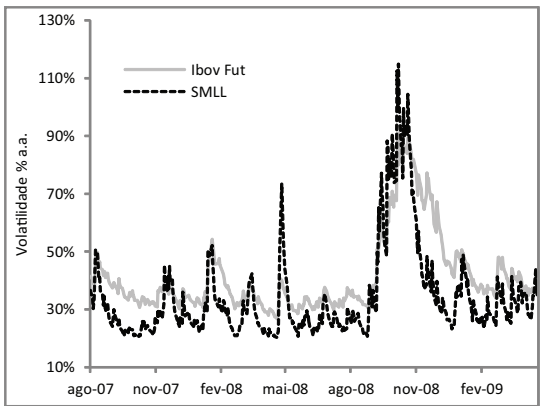

(f) SMLL

Figura 3 - Evolução da volatilidade dos índices do mercado à vista em relação ao IBOVESPA futuro entre agosto de 2007 e abril de 2009

Pode-se ainda acrescentar que os índices IBOVESPA, IBrX-50, IGC e MLCX, segundo a Figura 3, apresentaram um padrão de volatilidade muito próximo do IBOVESPA Futuro, indicando um comportamento similar neste período. O Índice FGV-100 e, principalmente, o índice SMLL, apresentaram um movimento menos volátil que o 
mercado futuro nos períodos pré e pós-crise. Contudo, no auge da crise, tanto o mercado spot (FGV-100 e SMLL) quanto o futuro apresentaram comportamento semelhante.

Determinado os padrões de volatilidade, é possível verificar a proposição de que o mercado futuro desestabiliza o mercado à vista, no sentido de aumentar a volatilidade nesse mercado durante a crise do subprime. A Tabela 4 apresenta os resultados do teste de causalidade proposto por Cheung e Ng (1996), baseado na função de correlação cruzada do quadrado dos resíduos padronizados, obtidos pelos modelos GARCH univariados.

O teste aplicado neste trabalho avaliou a relação causal, em termos de variância, entre cada índice do mercado à vista e o Ibovespa Futuro, sob a hipótese nula de não causalidade. Com base na Tabela 4, verifica-se que as estatísticas para o teste baseado na FCC não foram significativas. Portanto, conclui-se que não houve correlação em períodos diferentes entre as variáveis consideradas e, apesar de uma forte correlação contemporânea entre os mercados em questão, não se observou a causalidade na variância, no sentido de WeinerGranger, do mercado futuro em relação ao mercado spot. Em termos do teste proposto por Cheung e Ng (1996), não se rejeitou a hipótese de não causalidade entre as volatilidades dos indicadores do mercado acionário à vista e futuro brasileiros. 
Tabela 4 - Teste de causalidade baseado na FCC entre as volatilidades do mercado à vista e futuro

\begin{tabular}{|c|c|c|c|c|c|c|}
\hline & \multicolumn{3}{|c|}{ IBOVESPA } & \multicolumn{3}{|c|}{ FGV-100 } \\
\hline Defasagem & $r_{u v}(k)$ & $\mathrm{p}$-valor $\left(\chi_{k}^{2}\right)$ & $\begin{array}{l}\text { p-valor } \\
(N(0,1))\end{array}$ & $r_{u v}(k)$ & p-valor $\left(\chi_{k}^{2}\right)$ & $\begin{array}{l}\text { p-valor } \\
(N(0,1))\end{array}$ \\
\hline 5 & 0,0664 & 0,112 & 0,911 & 0,0211 & 0,160 & 0,3336 \\
\hline 4 & 0,0577 & 0,284 & 0,880 & 0,1112 & 0,121 & 0,127 \\
\hline 3 & $-0,0472$ & 0,572 & 0,167 & $-0,0608$ & 0,235 & 0,893 \\
\hline 2 & 0,0193 & 0,914 & 0,653 & 0,0307 & 0,509 & 0,265 \\
\hline 1 & $-0,0072$ & 0,989 & 0,441 & $-0,0447$ & 0,621 & 0,835 \\
\hline 0 & 0,904 & - & 0,000 & 0,8633 & - & 0,000 \\
\hline-1 & $-0,0413$ & 0,698 & 0,198 & $-0,0306$ & 0,821 & 0,734 \\
\hline-2 & 0,0539 & 0,379 & 0,864 & 0,0651 & 0,337 & 0,158 \\
\hline-3 & $-0,0176$ & 0,356 & 0,359 & 0,0274 & 0,288 & 0,712 \\
\hline-4 & 0,0240 & 0,315 & 0,687 & 0,0436 & 0,193 & 0,187 \\
\hline \multirow[t]{2}{*}{-5} & 0,0556 & 0,164 & 0,871 & 0,0308 & 0,158 & 0,265 \\
\hline & \multicolumn{3}{|c|}{$\mathrm{IBrX}-50$} & \multicolumn{3}{|c|}{ IGC } \\
\hline Defasagem & $r_{u v}(k)$ & p-valor $\left(\chi_{k}^{2}\right)$ & $\begin{array}{c}\text { p-valor } \\
(N(0,1))\end{array}$ & $r_{u v}(k)$ & $\begin{array}{c}\text { p-valor } \\
\left(\chi_{k}^{2}\right)\end{array}$ & $\begin{array}{l}\text { p-valor } \\
(N(0,1))\end{array}$ \\
\hline 5 & 0,0310 & 0,190 & 0,264 & 0,0336 & 0,102 & 0,247 \\
\hline 4 & 0,0607 & 0,233 & 0,109 & 0,0766 & 0,129 & 0,598 \\
\hline 3 & $-0,0445$ & 0,505 & 0,818 & $-0,0470$ & 0,443 & 0,831 \\
\hline 2 & 0,0307 & 0,766 & 0,350 & 0,0392 & 0,039 & 0,705 \\
\hline 1 & $-0,0476$ & 0,824 & 0,732 & $-0,0113$ & 0,973 & 0,592 \\
\hline 0 & 0,8659 & - & 0,000 & 0,947 & - & 0,000 \\
\hline-1 & $-0,0377$ & 0,741 & 0,780 & $-0,0427$ & 0,681 & 0,809 \\
\hline-2 & 0,0712 & 0,255 & 0,730 & 0,050 & 0,399 & 0,151 \\
\hline-3 & $-0,0236$ & 0,227 & 0,685 & $-0,0062$ & 0,396 & 0,550 \\
\hline-4 & 0,0335 & 0,174 & 0,235 & 0,0279 & 0,336 & 0,284 \\
\hline \multirow[t]{2}{*}{-5} & 0,0444 & 0,115 & 0,183 & 0,0191 & 0,311 & 0,348 \\
\hline & \multicolumn{3}{|c|}{ SMLL } & \multicolumn{3}{|c|}{ MLCX } \\
\hline Defasagem & $r_{u v}(k)$ & $p$-valor $\left(\chi_{k}^{2}\right)$ & $\begin{array}{c}\text { p-valor } \\
(N(0,1))\end{array}$ & $r_{u v}(k)$ & $\begin{array}{c}\text { p-valor } \\
\left(\chi_{k}^{2}\right)\end{array}$ & $\begin{array}{c}\text { p-valor } \\
(N(0,1))\end{array}$ \\
\hline 5 & 0,0246 & 0,180 & 0,308 & 0,0332 & 0,169 & 0,249 \\
\hline 4 & 0,0500 & 0,190 & 0,154 & 0,0629 & 0,214 & 0,139 \\
\hline 3 & $-0,0805$ & 0,158 & 0,950 & $-0,0444$ & 0,491 & 0,818 \\
\hline 2 & 0,0394 & 0,617 & 0,210 & 0,0199 & 0,742 & 0,342 \\
\hline 1 & 0,0272 & 0,856 & 0,289 & $-0,0320$ & 0,806 & 0,744 \\
\hline 0 & 0,9594 & - & 0,000 & 0,8725 & - & 0,000 \\
\hline-1 & $-0,0181$ & 0,933 & 0,645 & $-0,0400$ & 0,714 & 0,794 \\
\hline-2 & 0,0328 & 0,744 & 0,251 & 0,0733 & 0,231 & 0,670 \\
\hline-3 & 0,0021 & 0,743 & 0,482 & $-0,0245$ & 0,203 & 0,692 \\
\hline-4 & 0,0145 & 0,711 & 0,384 & 0,0340 & 0,195 & 0,244 \\
\hline-5 & 0,0415 & 0,495 & 0,199 & 0,0437 & 0,107 & 0,187 \\
\hline
\end{tabular}

Para confrontar tais resultados, a Tabela 5 apresenta a aplicação do teste LM, proposto por Hafner e Herwartz (2006), baseado no multiplicador de Lagrange. Testou-se a hipótese nula de não causalidade e, de acordo com os $p$-valores obtidos, aceitou-se tal hipótese. Dessa forma, constata-se que, durante a crise do subprime, não se verificou uma desestabilização ou incremento da volatilidade do mercado à vista, decorrente das oscilações no mercado futuro. 
Tabela 5 - Teste de causalidade LM para as volatilidades do mercado à vista $\mathrm{e}$ futuro

\begin{tabular}{l|cccccc}
\hline & \multicolumn{6}{|c}{ Índices do mercado à vista } \\
\hline & IBOVESPA & FGV-100 & IBrX-50 & IGC & SMLL & MLCX \\
\hline$\lambda_{\text {LM }}$ & 2,8616 & 3,4719 & 2,9222 & 3,8238 & 1,9880 & 3,1241 \\
p-valor $\left(\chi_{2}^{2}\right)$ & 0,2393 & 0,1764 & 0,2322 & 0,1480 & 0,3697 & 0,2101 \\
\hline
\end{tabular}

Os resultados confirmam o que, em geral, observa-se na literatura sobre o tema, no sentido de que não há evidências empíricas para afirmar a assertiva de desestabilização no mercado à vista motivada pelos mercados futuros. Vale observar que Galvão et al. (2000) obtiveram conclusão similar ao estudar a causalidade entre a volatilidade dos preços futuros e spot do Ibovespa no Brasil entre julho de 1994 e agosto de 1995, período marcado pela sequência de forte entrada de capitais estrangeiros (até dezembro de 1994) e posterior saída devido à crise do México.

\section{Conclusões}

A estabilidade e o crescimento econômico da economia brasileira ao longo da década de 2000, somados à grande entrada de capital estrangeiro no mercado financeiro, permitiram um considerável incremento no volume transacionado de derivativos no País. Mesmo com o objetivo de repartição dos riscos entre os agentes e diminuição do grau de incerteza, a literatura tem investigado se esses instrumentos, sobretudo o mercado futuro, desestabilizam o mercado à vista, no sentido de alterar seu padrão de volatilidade, desencadeando oscilações capazes de elevar o risco do sistema financeiro em geral, principalmente em períodos de crises.

No cenário econômico recente, a crise do subprime, originária no mercado norte-americano que, posteriormente, tomou proporções sistêmicas, chamou grande atenção, devido a sua magnitude. Dessa forma, o presente trabalho avaliou, no período desta crise financeira, a hipótese de causalidade na variância entre o mercado acionário à vista e futuro brasileiro, no sentido de que o último desestabiliza o primeiro. 
Os resultados indicaram que o mercado de ações brasileiro absorveu as consequências da crise, sendo verificado um forte aumento na volatilidade dos índices selecionados, devido às notícias da estatização das instituições Fannie Mae e Freddie Mac e ao pedido de concordata do Lehman Brothers, que iniciaram o momento crítico da crise no Brasil. Os resultados dos testes de causalidade, baseados na função de correlação cruzada e no multiplicador lagrangeano, apontaram para a não rejeição da hipótese de não causalidade na variância entre os mercados à vista e futuro. Com isso, não se pode inferir que, durante a crise do subprime, os movimentos do mercado futuro ajudaram a desestabilizar o mercado à vista, no sentido de aumentar sua volatilidade e, por conseguinte, o risco. Assim, nesse período, as instabilidades exógenas, i.e. não decorrentes do mercado futuro, mostraram-se como mais relevantes para explicar as oscilações verificadas nos mercados spot.

Trabalhos futuros podem incluir a avaliação da causalidade por meio da estimação da variância com modelos multivariados, para se testar as hipóteses em termos da significância dos parâmetros estimados.

\section{Referências}

ACWORTH, W. Annual volume survey: 2010 record volume. Futures Industry Association, 2011.

AKAIKE, H. Maximum likelihood identification of Gaussian autoregressive moving average models. Biometrika, v. 60, p. 255-265, 1973.

ALEXAKIS, P. On the effect of index futures trading on stock market volatility. International Research Journal of Finance and Economics, v. 11, p. 7-20, 2007.

ALEXANDER, C. Pratical Financial Econometrics. Editora John Wiley, 2008, 426p.

ALEXANDER, C.; LAZAR, E. Normal mixture GARCH (1,1): Applications to exchange rate modeling. Journal of Applied Econometrics, v. 21, p. 307-336, 2006.

ANTONIOU, A.; HOLMES, P.; PRIESLEY, R. The effects on stock index futures trading on stock index volatility: an analysis of the asymmetric response of volatility to news. The Journal of Futures Markets, v. 18, n. 2, p. 151-166, 1998.

BAE, S. C.; KWON, T. H.; PARK, J. W. Derivatives trading, volatility spillover, and regulation: evidence from the Korean securities markets. The Journal of Futures Markets, v. 29, n. 6, p. 563-597, 2009.

BANDIVADEKAR, S.; GHOSH, S. Derivatives and volatility on Indian stock markets. Reserve Bank of India Occasional Papers, v. 24, n. 3, p. 187-201, 2003.

BANK FOR INTERNATIONAL SETTLEMENTS. Triennial and semiannual surveys - Positions in global over-the-counter (OTC) derivatives markets at end-June 2010. Bank for International Settlements, Monetary and Economic Department, 2010.

BOLLERSLEV, T. Generalized Autoregressive Conditional Heteroskedasticity. Journal of Econometrics, v. 31 , p. $303-327,1986$. 
BOLOGNA, P.; CAVALLO, L. Does the introduction of stock index futures effectively reduce stock market volatility? Is the futures effect immediate? Evidence from the Italian stock exchange using Garch. Applied Financial Economics, v. 12, n. 2, p. 183-192, 2002.

CHEUNG, Y. W.; NG, L. K. A causality in variance test and its application to financial market prices. Journal of Econometrics, v. 72, p. 33-48, 1996.

CHIANG, M. H.; WANG, Y. C. The impact of futures trading on spot index volatility: evidence from Taiwan index futures. Applied Economics Letters, v. 9, n. 6, p. 381-385, 2002.

COX, C. C. Futures trading and market information. Journal of Political Economy, v. 6, n. 84, p. 1215-1237, 1976.

DAWSON, P.; STAIKOUPAS, S. K. The impact of volatility derivatives on S\&P500 volatility. The Journal of Futures Markets, v. 12, n. 29, p. 1190-1213, 2009.

DEBASISH, S. S. Effect of futures trading on spot-price volatility: evidence for NSE Nifty using GARCH. The Journal of Risk Finance, v. 10, n. 1, p. 66-77, 2009.

DRAKOS, A. A.; KOURETAS, G. P.; ZARANGAS, L. P. Forecasting financial volatility of the Athens stock Exchange daily returns: An application of the asymmetric normal mixture GARCH models. International Journal of Finance and Economics, v. 15, p. 331-350, 2010.

DRIMBETAS, E.; SARIANNIDIS, N.; PORFIRIS, N. The effect of derivatives trading on volatility of the underlying asset: evidence from the Greek stock market. Applied Financial Economics, v. 17, n. 2, p. 139-148, 2007.

ENGLE, R. F. Autoregressive conditional heteroskedasticity with estimates of the variance of United Kingdom inflation. Econometrica, v. 50, n. 4, p. 987-1007, 1982.

ENGLE, R. F. New frontiers for ARCH models. Journal of Applied Econometrics, v. 17, p. 425-446, 2002.

FIGLEWSKI, S. Futures trading and volatility in the GNMA market. Journal of Finance, v. 36, p. 445-456, 1981.

FIA. Annual volume survey: 2010 record volume. Futures Industry Association, 2011.

GALVÃO, A. B. C.; PORTUGAL, M. S.; RIBEIRO, E. P. Volatilidade e Causalidade: Evidência para os mercados à vista e futuro de câmbio e índice de ações no Brasil. Revista Brasileira de Economia, v. 54 , n. 1 , p. $39-56,2000$.

GRANGER, C. W. J. Investigating Causal Relations by Econometric Models and Cross-Spectral Methods. Econometrica, v. 34, p. 424-438, 1969.

HAFNER, C. M.; HERWARTZ, H. A Lagrange multiplier test for causality in variance. Economics Letters, v. 93, p. 137-141, 2006.

HANNAN, E. J.; QUINN, B. G. The determination of the order of an autoregression. Journal of the Royal Statistical Society, v. 41, p. 190-195, 1979.

HONG, Y. A test for volatility spillover with application to exchange rates. Journal of Econometrics, v. 103, p. 183-224, 2001.

ILLUECA, M.; LAFUENTE, J. A. The effect of spot and futures trading on stock index market volatility: a nonparametric approach. The Journal of Futures Markets, v. 23, n. 9, p. 841-858, 2003.

ILLUECA, M.; LAFUENTE, J. A. Introducing the mini-futures contract on Ibex 35: implications for price discovery and volatility transmission. Spanish Economic Review, v. 10, n. 3, p. 197-219, 2008.

KASMAN, A.; KASMAN, S. The impact of futures trading on volatility of the underlying asset in the Turkish stock market. Physica A: Statistical Mechanics and its Applications, v. 387, n. 12, p. 2837-2845, 2008.

KNIGHT, J.; SATCHEL, S. (Eds.) Forecasting Volatility in the Financial Markets. 3 ed. Editora Elsevier, 2007.

LAURENT, S.; PETERS, J. P. G@RCH 2.2, an Ox package for estimating and forecasting various ARCH models. Journal of Economic Surveys, v. 16, p. 447-485, 2002.

Est. Econ., São Paulo, vol. 42, n.4, p. 801-825, out.-dez. 2012 
MAYEW, S. The impact of derivatives on cash markets: what have we learned? Working paper, Department of Banking and Finance, Terry College of Business, University of Georgia, 2000.

MORETTIN, P. A.; TOLOI, C. M. C. Análise de Séries Temporais. Editora Edgard Blücher, 2006.

PILAR, C.; RAFAEL, S. Does derivatives trading destabilize the underlying assets? Evidence from the Spanish stock market. Applied Economics Letters, v. 9, n. 2, p. 107-110, 2002.

POON, S-H.; GRANGER, C. Forecasting volatility in financial markets: A review. Journal of Economic Literature, v. XLI, p. 478-539, 2003.

POW, W. C.; POSHAKWALE, S. The impact of futures contracts on the spot market volatility: the case of Kuala Lumpur stock exchange. Applied Financial Economics, v. 14, n. 2, p. 143-154, 2004.

RAO, R. Impact of financial derivative products on spot market volatility: a study of nifty. The ICFAI Journal of Derivatives Market, v. 4, n. 1, p. 7-16, 2007.

RYOO, H. J.; SMITH, G. The impact of stock index futures on the Korean stock market. Applied Financial Economics, v. 14, n. 4, p. 243-251, 2004.

SCHWARZ, G. Estimating the dimension of a model. Annals of Statistics, v. 6, p.461-464, 1978.

SPYROU, S. I. Index futures trading and spot price volatility. Journal of Emerging Market Finance, v. 4, n. 2, p. 151-167, 2005.

SRINVASAN, P.; BHAT, K. S. The impact of futures trading on the spot market volatility of selected commercial banks in India. European Journal of Economics, Finance and Administrative Sciences, v. 14, p. 29-41, 2008.

STEIN, J. Informational externalities and welfare-reducing speculation. Journal of Political Economy, v. 95, p. 1123-1145, 1987.

TRIPATHY, N.; RAO, S. V. R.; KANAGARA, A. Impact of derivatives trading on spot market volatility: an empirical study. International Journal of Applied Decision Sciences, v. 2, n. 2, p. 209-232, 2005.

YO, S. W. Index futures trading and spot price volatility. Applied Economics Letters, v. 8, n. 3, p. 183$186,2001$.

ZHONG, M.; DARRAT, A. F.; OTERO, R. Price discovery and volatility spillovers in index futures markets: some evidence from Mexico. Journal of Banking \& Finance, v. 28, n. 12, p. 3037-3054, 2004. 